\title{
Manual Aspiration Thrombectomy in Acute Myocardial Infarction: A Clinical Experience
}

\author{
Abhishek Jaiswal, MD ${ }^{1}$ Simcha Pollack, $\mathrm{PhD}^{2}$ Astha Chichra, $\mathrm{MD}^{3} \quad$ Emmanuel Moustakakis, MD \\ Chong Park, MD ${ }^{4}$ Todd Kerwin, $\mathrm{MD}^{4}$
}

1 Tulane University Heart and Vascular Institute, New Orleans, Louisiana

2 Department of Computer Information Systems and Decision Sciences, St. Johns University, New York

${ }^{3}$ Department of Internal Medicine, Hofstra North Shore-LIJ Health System, Manhasset, New York

${ }^{4}$ Division of Cardiology, New York Hospital Queens/Weill Medical College of Cornell University, New York

Address for correspondence Abhishek Jaiswal, MD, 1430 Tulane Avenue, SL-48, Tulane University Heart and Vascular Institute, New Orleans, LA 70112 (e-mail: jais.abhishek@gmail.com).

Int J Angiol 2016;25:20-28.

\begin{abstract}
Keywords

- acute myocardial infarction

- angioplasty

- atherosclerosis

- infarction

- myocardial infarction

- thrombectomy

Multiple clinical studies have failed to establish the role of routine use of thrombectomy in ST-elevation myocardial infarction (STEMI) patients. There is a paucity of data on the impact of thrombectomy in unselected STEMI patients outside clinical trials. We sought to evaluate the clinical variables and outcomes associated with the performance of thrombectomy in STEMI patients. We retrospectively examined the clinical outcomes in all STEMI patients who underwent successful percutaneous intervention $(\mathrm{PCI})$ at our center. Patients were divided into two groups, one with patients who underwent conventional $\mathrm{PCl}$ and another with patients who had thrombus aspiration in addition to conventional $\mathrm{PCl}$. We compared the baseline clinical characteristics, laboratory investigations, re-infarction rates, and all-cause mortality. Total 477 consecutive STEMI patients were identified. Overall, $29 \%$ (139) of the patients underwent conventional $\mathrm{PCl}$ and $71 \%$ (338) of the patients were treated with aspiration thrombectomy and PCI. In addition to the presence of thrombus, patients with nonanterior infarction, and patients with hemodynamic instability requiring intra-aortic balloon pump support were more likely to undergo thrombectomy. Thrombectomy was associated with higher enzymatic infarction (creatine kinase: 2,796 [2,575] vs. 1,716 [1,662]; $p<0.0001$; CKMB: 210.6 [156.0] vs. 142.0 [121.9], $p<0.0001)$. However, thrombectomy was not associated with any difference in 30 day reinfarction rate $(3.3$ vs. $2.9 \%, p=0.83)$, mortality ( 5.0 vs. $7.2 \%, p=0.35$ ), or composite of death and 30 day reinfarction ( 7.7 vs. $9.4 \%, p=0.55)$. We observed that STEMI patients with anterior infarction and hemodynamic instability were more likely to undergo thrombectomy during primary $\mathrm{PCl}$.
\end{abstract}

The outcome of patients with ST-elevation myocardial infarction (STEMI) has significantly improved in the reperfusion era. One of the biggest challenges faced during percutaneous intervention (PCI) of STEMI patients is distal embolization of thrombus, limiting myocardial reperfusion and adversely affecting clinical outcomes. ${ }^{1,2}$ As a result, there has been a growing interest in the idea of thrombus removal to improve the outcomes. The TAPAS (Thrombus Aspiration during published online March 23, 2015
Copyright @ 2016 by Thieme Medical Publishers, Inc., 333 Seventh Avenue, New York, NY 10001, USA. Tel: +1(212) 584-4662.
DOI http://dx.doi.org/ 10.1055/s-0035-1547515. ISSN 1061-1711. 
Percutaneous coronary intervention in Acute myocardial infarction Study) trial was the landmark study, which established the role of thrombus aspiration in reducing no-reflow, improving myocardial blush (MB), ST-segment resolution and mortality at 1 year. ${ }^{3,4}$ However, subsequent studies have shown conflicting results. ${ }^{5-11}$ We sought to evaluate the clinical variables and outcomes associated with the performance of thrombectomy in STEMI patients.

\section{Methods}

\section{Data and Clinical Definitions}

Patients included in this study were evaluated from a singlecenter STEMI database/registry. This registry was developed as part of a quality improvement and outcome monitoring device. We defined STEMI to be present if the initial electrocardiogram (EKG) showed at least 1-mm ST-segment elevation (> 2-mm ST-segment elevation in right precordial leads, V1-V3) in two or more contiguous leads in standard EKG at the time of the emergency room arrival or in the EKG done by paramedics; in conjunction with ischemic symptoms. All patients who undergo $\mathrm{PCI}$ are entered into this prospective registry. Standardized information is collected which includes demographic profile, clinical characteristics, ischemic time, angiographic characteristics and procedural details, laboratory data, hospitalization course, and subsequent clinical follow-up. Ethnicity is self-identified and is finally determined in conjunction with the country of origin. All of these patients routinely receive $325 \mathrm{mg}$ aspirin, $600 \mathrm{mg}$ of clopidogrel, and $80 \mathrm{mg}$ of atorvastatin in the emergency room. Cardiac enzymes were drawn at the time of presentation in the emergency room and subsequently in 6 to 8 hour intervals. Due to the change in hospital's protocol for checking troponin I to troponin T we only report creatine kinase (CK) and CK-MB fraction. Guideline-directed medical therapy is prescribed for all patients, including 81to $325 \mathrm{mg}$ aspirin, $75 \mathrm{mg}$ clopidogrel, and statins, $\beta$ blocker, and angiotensinconverting enzyme inhibitor. Patients are routinely contacted 30 days after the discharge. Clinical outcomes are ascertained via telephonic contact with the patient, family, or physician. In addition, the social security death index is reviewed for mortality detection and confirmation. Predischarge left ventricular function (LVEF) is determined by a follow-up transthoracic echocardiogram (done between 3 and 7 days) in all patients. The LVEF is determined using the Simpson biplane formula, according to the recommendations of the American Society of Echocardiography.

$\mathrm{PCI}$ is performed according to the best recommended contemporary clinical practice. All patients received bivalirudin during the procedure. The decision on the adjunctive use of glycoprotein IIb/IIla inhibitors, intra-aortic balloon pump (IBMP), and mode of revascularization: surgical versus percutaneous, bare metal versus drug eluting is at the discretion of the treating interventional cardiologist. The thrombolysis in myocardial infarction (TIMI) grade flow is defined as follows: TIMI 0 flow (no perfusion): absence of any antegrade flow beyond a coronary occlusion; TIMI 1 flow (penetration without perfusion): faint antegrade coronary flow beyond the occlusion, with incomplete filling of the distal coronary bed; TIMI 2 flow (partial reperfusion): delayed antegrade flow with complete filling of the distal territory; and TIMI 3 flow (complete perfusion): normal flow which fills the distal coronary bed completely. MB was graded as described by Van't Hof et $\mathrm{al}^{12}$ : Grade 0 to 1 was minimal to no $\mathrm{MB}$ or contrast density (relative to the dye density in the uninvolved areas); grade 2 was moderate MB; and grade 3 was normal MB. Persistent MB is considered grade 0 . Thrombectomy was performed using either a Fetch (Possis Medical Inc., Minneapolis, MN) or an Export catheter (Medtronic Inc., Minneapolis, $\mathrm{MN}$ ) before balloon dilation.

\section{Study Subjects}

Our STEMI registry was retrospectively accessed and data were extracted on patients who underwent coronary angiography for STEMI management between August 2008 and November 2012. Patients were excluded if they did not undergo angiography, underwent surgical revascularization, failed revascularization, or died before completion of revascularization.

\section{Clinical Outcomes/Endpoints}

We compared postrevascularization TIMI grade flow in the culprit epicardial vessels, MB score, peak serum cardiac enzyme level, resolution of the elevated ST segments on EKG, 30 day reinfarction, and all-cause mortality between the groups. We defined complete revascularization as grade 3 TIMI flow with a MB score of 2 or greater. EKGs were assessed for ST-segment resolution within 90 minutes after PCI was performed. We defined early complete resolution if ST segments went down by $>70 \%$ as compared with the presentation EKG.

\section{Statistical Methods}

Continuous variables are presented as mean $( \pm$ standard deviation) or, median and interquartile range (IQR) as appropriate, and categorical variables as a percentage. Standard tests were done to check if the data were normally distributed. Continuous data were compared using unpaired Student $t$-test or Mann-Whitney U test as appropriate. Categorical data were compared using Pearson chi-square test. A multivariate analysis using forward stepwise logistic regression was done to check for independent predictors of thrombectomy and clinical outcomes-peak serum CPK, peak serum CK-MB, all-cause mortality, 30-day reinfarction, and a composite of death and mortality. For the multiple regression analyses, univariate analysis was used to select for those factors showing $p<0.1$. A $p$-value of 0.05 was considered significant in both univariate and multivariate analyses. No adjustment was done for multiple analyses. Survival rates were calculated using the Kaplan-Meier method, and differences were tested with the log-rank test. The independent effects of variables on prognosis were calculated using a Cox proportional hazards regression model. Odds ratios (ORs) are presented with their 95\% confidence intervals (CIs). Statistical analysis was done using SAS software 9.3 (SAS Institute Inc., Cary, NC) for Windows. 


\section{Results}

\section{Baseline Characteristics}

A total of 477 patients were identified. Overall, 29\% (139) of the patients underwent conventional PCI, whereas 71\% (338) of the patients underwent thrombectomy with PCI. Baseline demographics, clinical characteristics, and angiographic findings are described in -Table 1. Patients who underwent thrombectomy, compared with conventional PCI, were younger (61.1 [13.3] vs. 64.1 [12.6] years, $p=0.03$ ), more likely to present with cardiogenic shock, unstable sustained ventricular arrhythmia requiring electrical defibrillation, thrombotic occlusion of the infarct related artery, and more often required IABP support. We observed an increased utilization of glycoprotein IIB/IIIA inhibitors (21.6 vs. $12.9 \%, p=0.03$ ) in patients who underwent thrombectomy. Thrombectomy group patients presented with lower left ventricular systolic function as assessed by ventriculography done during diagnostic angiography. In the thrombectomy group there was a trend toward fewer epicardial vessels with significant steno$\operatorname{sis}(1.8$ [0.9] vs. 1.9 [0.9], $p=0.09$ ) and lower $\operatorname{LVEF}(49.6$ [11.9] vs. $51.8[11.1] \%, p=0.07)$ by echocardiogram done at the time of hospital discharge (-Table $\mathbf{1}$ ).

\section{Predictors of Thrombectomy}

On multivariate analysis anterior infarction (OR: $1.7,95 \% \mathrm{CI}$ : [1.1-2.8], $p=0.03$ ), preprocedural TIMI flow $0 / 1$ or occluded artery (OR: 8.6, 95\% CI: [4.5-16.4], $p<0.0001$ ), and use of IABP support (OR: 0.4, 95\% CI: [0.11-1], $p=0.06$ ) were found to be independent predictors of thrombectomy usage in addition to the presence of thrombus (-Table $\mathbf{2}$ ).

\section{Clinical Outcomes and Endpoints}

\section{Enzymatic Infarct Size}

Patients who underwent thrombectomy had higher peak serum cardiac enzymes (CPK: 2,796 [2,575] vs. 1,716 [1622], $p<0.0001$; CK-MB: 210.6 [156.0] vs. 142.2 [121.8], $p<0.0001$ ). The study sample was divided into two groups, those above and below the median value of the peak CPK $(1,740 \mathrm{IU} / \mathrm{ng})$. A second dichotomous grouping was defined by the median of CK-MB (158.1 IU/ng) levels. Age, initial LVEF, and thrombectomy were independent predictors of enzymatic infarct size as measured by CPK and CK-MB (- Table 3).

\section{ST-Segment Resolution}

The two groups did not differ with respect to early ST-segment resolution (thrombectomy: $72 \%$ vs. $\mathrm{PCI}: 67 \%, p=0.28$ ) and delayed ST-segment resolution (thrombectomy: $90 \%$ vs. PCI: $86 \%, p=0.21$ ). Modeling the time to ST-segment resolution via Cox proportional hazard regression showed that anterior infarction (hazard ratio [HR]: $0.795 \% \mathrm{CI}$ : [0.6-0.9], $p=0.014$ ), thrombectomy (HR: 1.6, 95\% CI: [1.3-2.1], $p=0.0002$ ) and glycoprotein IIB/IIIA use (HR: 0.6, 95\% CI: [0.4-0.8], $p=0.0003$ ) were independently associated with early STsegment resolution. Myocardial infarction: 30 day reinfarction rates were similar between the groups with $2.8 \%$ in conventional PCI group and $3.2 \%$ in thrombectomy group $(p=0.83)$.

\section{Mortality}

Overall, 10 patients died during follow-up in the conventional PCI group versus 17 patients in the thrombectomy group (-Table 4). Mortality rates were similar (5.0 vs. $7.2 \%$, $p=0.35$ ). Age (HR: 1.06, 95\% Cl: [1.0-1.1], $p=0.006)$ and symptom to balloon time (HR: 0.97, 95\% CI: [0.96-0.98], $p<0.0001)$ were the only independent predictors of allcause mortality. IABP support (HR: $4.2(0.8-21.9), p=0.08$ ) showed trends toward independent association with mortality. Kaplan-Meier analysis showing the survival based on allcause mortality is presented in - Fig. $\mathbf{1}$.

\section{Death or Myocardial Infarction}

Composite of all-cause mortality or 30 day re-infarction were similar in both groups (7.7\% versus $9.4 \%, p=0.55)$. Age (HR: 1.04, 95\% CI: [1.01-1.07], $p=0.004$ ), diabetes (HR: 2.0, 95\% CI: [1.0-4.1], $p=0.05$ ) and symptom to balloon time (HR: $0.99,95 \% \mathrm{CI}$ : [0.98-0.99], $p=0.015$ ) predicted the composite of death or re-infarction. Severity of CAD as determined by the number of vessels involved (HR: 1.5 [0.94-2.35], $p=0.09$ ) showed a trend toward association with the composite of death or myocardial infarction.

\section{Discussion}

The present study is one of the few studies ${ }^{13-17}$ which describe the role of routine thrombectomy on enzymatic infarction size, reinfarction, and mortality in a relatively large cohort of unselected STEMI patients who underwent PCI. In our retrospective cohort, we observed that patients with anterior wall myocardial infarction and hemodynamic instability were more likely to undergo mechanical thrombectomy during primary PCI. The mortality and re-infarction rates were comparable between the group who underwent thrombus aspiration and those who underwent conventional primary PCI. We also observed that patients who received thrombectomy sustained larger enzymatic infarction size and had less early ST segment resolution. Concurrent with existing literature, clinical outcomes in our STEMI cohort was influenced by age, presence of diabetes mellitus and ischemic duration.

In STEMI patients, mechanical reperfusion or primary $\mathrm{PCI}$ leads to rapid restoration of epicardial flow, which has been associated with improved clinical outcomes including survival. ${ }^{18,19}$ However, the patency of epicardial flow does not necessarily indicate adequate myocardial perfusion. This could be explained by microvascular occlusion due to distal embolization of atherothrombotic material during mechanical reperfusion. ${ }^{20}$ As many as $30 \%$ patients treated with primary percutaneous transluminal coronary angioplasty were found to have no reflow phenomenon jeopardizing myocardial perfusion. Since then various studies and clinical trials have been undertaken to look at different approaches to decrease distal embolization. Mainly based on the results from the TAPAS trial, ${ }^{3}$ ACC/AHA/ESC ${ }^{18,19}$ advocate use of routine thrombectomy to protect from distal embolization during PCI for STEMI. ${ }^{21}$ The findings from TAPAS were replicated in the EXPIRA (thrombectomy with EXPort 
Table 1 Baseline, angiographic, laboratory, and outcome characteristics as classified by thrombectomy during PCl in patients with ST-elevation myocardial infarction

\begin{tabular}{|c|c|c|c|}
\hline \multirow[t]{2}{*}{ Variable (mean [SD] or $n[\%]$ ) } & \multicolumn{2}{|c|}{ Thrombectomy } & \multirow[t]{2}{*}{$p$-Value ${ }^{a}$} \\
\hline & No (139) & Yes (337) & \\
\hline \multicolumn{4}{|l|}{ Baseline characteristics } \\
\hline Age & $64.0(12.6)$ & $61.1(13.3)$ & 0.03 \\
\hline Male $n(\%)$ & 109 (78.4\%) & $272(80.5 \%)$ & 0.61 \\
\hline BMI & $27.2(5.3)$ & $27.3(5.6)$ & 0.81 \\
\hline Diabetes & $47(33.1 \%)$ & $95(28.1 \%)$ & 0.22 \\
\hline Hypertension & $97(69.8 \%)$ & $215(63.6 \%)$ & 0.20 \\
\hline Hyperlipidemia & $87(62.6 \%)$ & $208(61.5 \%)$ & 0.83 \\
\hline Current smoker & $41(29.5 \%)$ & $124(36.7 \%)$ & 0.13 \\
\hline Exsmoker & $31(22.3 \%)$ & $68(20.1 \%)$ & 0.59 \\
\hline Previous CAD & $32(23.0 \%)$ & $76(22.5 \%)$ & 0.90 \\
\hline Prior Ml & $11(7.9 \%)$ & $27(8.0 \%)$ & 0.98 \\
\hline Total ischemic time (median) (IQR) & $238(338.5)$ & $222.5(342.5)$ & 0.19 \\
\hline Cardiogenic shock & $7(5.0 \%)$ & $45(13.3 \%)$ & 0.008 \\
\hline Sustained VT/VF & $1(0.7 \%)$ & $22(6.5 \%)$ & 0.007 \\
\hline \multicolumn{4}{|l|}{ Angiographic characteristics } \\
\hline No. of vessels involved & $1.9(0.8)$ & $1.8(0.8)$ & 0.09 \\
\hline LAD infarction & $79(56.8 \%)$ & $158(46.7 \%)$ & 0.045 \\
\hline Preprocedural TIMI flow 0/1 & $88(63 \%)$ & $323(96 \%)$ & $<0.0001$ \\
\hline Thrombus & $102(73.4 \%)$ & $338(100 \%)$ & $<0.0001$ \\
\hline IIB/IIIA use & $18(12.9 \%)$ & $73(21.6 \%)$ & 0.03 \\
\hline Vasodilator & 105 (75.5\%) & $251(74.3 \%)$ & 0.77 \\
\hline LVEF at the time of angiogram & $47.5(10.3)$ & $44.1(11.2)$ & 0.003 \\
\hline LVEDP & $23.5(8.4)$ & $24.3(9.0)$ & 0.36 \\
\hline IABP support & $13(9.4 \%)$ & $86(25.5 \%)$ & $<0.0001$ \\
\hline Lesion stented & $136(97.8 \%)$ & 333 (98.5\%) & 0.60 \\
\hline LVEF at the time of discharge & $51.8(11.1)$ & $49.6(11.9)$ & 0.07 \\
\hline Postprocedural TIMI flow & $2.8(0.4)$ & $2.8(0.5)$ & 0.92 \\
\hline Myocardial blush grade & $2.4(0.8)$ & $2.5(0.8)$ & 0.50 \\
\hline \multicolumn{4}{|l|}{ Laboratory investigations } \\
\hline GFR-AD & $83.6(39.1)$ & $87.8(37.9)$ & 0.28 \\
\hline GFR-DC & $94.6(75.2)$ & $95.2(44.6)$ & 0.9371 \\
\hline CPK on admission (median) (IQR) & $167(260)$ & $160(289.25)$ & 0.40 \\
\hline Peak CPK (median) (IQR) & $1,061(1,922)$ & $2,049(2,695)$ & $<0.0001$ \\
\hline Peak CKMB (median) (IQR) & $108(192.4)$ & $182.5(217.75)$ & $<0.0001$ \\
\hline Early ST-segment resolution & $93(66.9 \%)$ & $243(71.9 \%)$ & 0.28 \\
\hline Delayed ST-segment resolution & $119(85.6 \%)$ & $303(89.6 \%)$ & 0.21 \\
\hline Death & $10(7.2 \%)$ & $17(5.0 \%)$ & 0.35 \\
\hline Myocardial infarction & $4(2.9 \%)$ & $11(3.3 \%)$ & 0.83 \\
\hline Composite MI or death & $13(9.4 \%)$ & $26(7.7 \%)$ & 0.55 \\
\hline
\end{tabular}

Abbreviations: BMI, body mass index; CAD, coronary artery disease; CK-MB, creatinine kinase myocardial brain fraction; CPK, creatinine phosphokinase; GFR$A D$, glomerular filtration rate at admission; GFR-DC, glomerular filtration rate at discharge; IABP, intra-aortic balloon pump; IIB/IIIA, glycoprotein IIB/IIA inhibitor; IQR, interquartile range; LAD, left anterior descending artery; LVEDP, left ventricular end diastolic pressure; LVEF, left ventricular ejection fraction; MI, myocardial infarction; SD, standard deviation; TIMI, thrombolysis in myocardial infarction; VT/VF, ventricular tachycardia/fibrillation.

${ }^{a} p$-Value generated by independent $t$-test for continuous variables and "exact" version of the chi-square test for categorical variables. 
24 Thrombus Aspiration in Acute Myocardial Infarction Jaiswal et al.

Table 2 Multivariate predictors of thrombectomy in ST-segment elevation myocardial infarction patients

\begin{tabular}{|l|l|l|l|}
\hline Variable & Odds ratio & $95 \%$ Confidence interval & $p$-Value \\
\hline Age & 1.01 & $0.997-1.032$ & 0.11 \\
\hline Cardiogenic shock & 1.4 & $0.385-5.318$ & 0.59 \\
\hline Sustained VT/VF & 0.3 & $0.03-2.04$ & 0.20 \\
\hline LAD infarction & 1.7 & $1.1-2.8$ & 0.03 \\
\hline Preprocedural TIMI flow 0/1 & 8.6 & $4.5-16.4$ & $<0.0001$ \\
\hline IIB/IIIA use & 0.7 & $0.4-1.3$ & 0.28 \\
\hline IABP support & 0.4 & $0.1-1.1$ & 0.06 \\
\hline Peak CPK & 1.0 & $1.0-1.0$ & 0.67 \\
\hline Peak CKMB & 0.998 & $0.995-1.000$ & 0.11 \\
\hline
\end{tabular}

Abbreviations: CK-MB, creatinine kinase myocardial brain fraction; CPK, creatinine phosphokinase; IABP, intra-aortic balloon pump; IIB/IIIA, glycoprotein IIB/ IIA inhibitor; LAD, left anterior descending artery; TIMI, thrombolysis in myocardial infarction; VT/VF, ventricular tachycardia/fibrillation.

catheter in Infarct-Related Artery during primary percutaneous coronary intervention) study which showed that the infarct size was reduced in patients who underwent manual thrombectomy. ${ }^{5}$ However, various single-center and multicenter studies have demonstrated diverse results. ${ }^{5,7,22-30}$ In addition, the strong evidence supporting the benefit of routine thrombectomy on angiographic outcomes, including TIMI grade flow, no-reflow, MB; and ST resolution did not translate well into supporting the mortality benefit. ${ }^{6}$ In a randomized trial Kaltoft et al concluded that routine

Table 3 Predictors of outcomes/endpoints after ST-segment elevation myocardial infarction on multivariate Cox proportional hazards time-to-event analysis

\begin{tabular}{|c|c|c|c|}
\hline Clinical outcome variables & Predictors & $\begin{array}{l}\text { Hazard ratio } \\
\text { (95\% confidence interval) }\end{array}$ & $p$-Value \\
\hline \multirow[t]{11}{*}{ Death } & Age & $1.061(1.018-1.107)$ & 0.006 \\
\hline & Diabetes & $1.808(0.759-4.303)$ & 0.19 \\
\hline & Ever smoked & $0.875(0.351-2.181)$ & 0.77 \\
\hline & Total ischemic duration & $0.970(0.958-0.983)$ & $<0.0001$ \\
\hline & Cardiogenic shock & $0.344(0.070-1.694)$ & 0.19 \\
\hline & IABP support & $4.243(0.822-21.897)$ & 0.08 \\
\hline & LVEF at the time of angiogram & $0.987(0.934-1.042)$ & 0.63 \\
\hline & Number of vessels involved & $1.501(0.787-2.863)$ & 0.22 \\
\hline & LAD infarction & $0.751(0.229-2.464)$ & 0.64 \\
\hline & Thrombectomy & $1.307(0.497-3.432)$ & 0.59 \\
\hline & IIB/IIIA use & $0.565(0.192-1.662)$ & 0.30 \\
\hline \multirow[t]{11}{*}{ Death or $30-\mathrm{d}$ myocardial reinfarction } & Age & $1.044(1.014-1.075)$ & 0.004 \\
\hline & Diabetes & $2.022(0.996-4.105)$ & 0.05 \\
\hline & Ever smoked & $0.848(0.412-1.747)$ & 0.66 \\
\hline & Total ischemic duration & 0.994 (0.989-0.999) & 0.015 \\
\hline & Cardiogenic shock & $0.859(0.223-3.312)$ & 0.82 \\
\hline & IABP support & $1.411(0.378-5.272)$ & 0.61 \\
\hline & LVEF at the time of angiogram & $0.997(0.955-1.041)$ & 0.89 \\
\hline & Number of vessels involved & $1.483(0.936-2.350)$ & 0.09 \\
\hline & LAD infarction & $0.812(0.338-1.948)$ & 0.64 \\
\hline & Thrombectomy & $1.921(0.902-4.091)$ & 0.09 \\
\hline & IIB/IIIA use & $0.662(0.286-1.535)$ & 0.34 \\
\hline
\end{tabular}


Table 3 (Continued)

\begin{tabular}{|c|c|c|c|}
\hline Clinical outcome variables & Predictors & $\begin{array}{l}\text { Hazard ratio } \\
\text { (95\% confidence interval) }\end{array}$ & $p$-Value \\
\hline \multirow[t]{11}{*}{ ST-segment resolution } & Age & $0.997(0.988-1.006)$ & 0.48 \\
\hline & Diabetes & $0.894(0.697-1.147)$ & 0.38 \\
\hline & Ever smoked & $0.986(0.777-1.251)$ & 0.91 \\
\hline & Total ischemic duration & $1.000(1.000-1.000)$ & 0.46 \\
\hline & Cardiogenic shock & $0.651(0.388-1.091)$ & 0.10 \\
\hline & IABP support & $1.227(0.813-1.851)$ & 0.33 \\
\hline & LVEF at the time of angiogram & $1.002(0.989-1.016)$ & 0.74 \\
\hline & Number of vessels involved & $0.997(0.859-1.157)$ & 0.97 \\
\hline & LAD infarction & $0.718(0.552-0.936)$ & 0.014 \\
\hline & Thrombectomy & $1.635(1.268-2.110)$ & 0.0002 \\
\hline & IIB/IIIA use & $0.581(0.432-0.782)$ & 0.0003 \\
\hline \multirow[t]{11}{*}{ Peak serum CK-MB } & Age & $0.989(0.978-1.000)$ & 0.05 \\
\hline & Diabetes & $0.788(0.582-1.068)$ & 0.12 \\
\hline & Ever smoked & $0.890(0.674-1.176)$ & 0.41 \\
\hline & Total ischemic duration & $1.000(1.000-1.000)$ & 0.07 \\
\hline & Cardiogenic shock & $0.743(0.463-1.191)$ & 0.22 \\
\hline & IABP support & $1.426(0.946-2.149)$ & 0.09 \\
\hline & LVEF at the time of angiogram & $0.966(0.951-0.981)$ & $<0.0001$ \\
\hline & Number of vessels involved & $1.016(0.852-1.211)$ & 0.86 \\
\hline & LAD infarction & $0.986(0.715-1.361)$ & 0.93 \\
\hline & Thrombectomy & $2.132(1.539-2.955)$ & $<0.0001$ \\
\hline & IIB/IIIA use & $0.845(0.616-1.161)$ & 0.30 \\
\hline \multirow[t]{11}{*}{ Peak serum CPK } & Age & $0.982(0.971-0.993)$ & 0.001 \\
\hline & Diabetes & $0.878(0.654-1.179)$ & 0.39 \\
\hline & Ever smoked & $0.795(0.603-1.048)$ & 0.10 \\
\hline & Total ischemic duration & $1.000(1.000-1.000)$ & 0.36 \\
\hline & Cardiogenic shock & $0.838(0.526-1.336)$ & 0.46 \\
\hline & IABP support & $1.403(0.928-2.119)$ & 0.11 \\
\hline & LVEF at the time of angiogram & $0.969(0.954-0.985)$ & $<0.0001$ \\
\hline & Number of vessels involved & $1.055(0.886-1.258)$ & 0.55 \\
\hline & LAD infarction & $1.202(0.871-1.658)$ & 0.26 \\
\hline & Thrombectomy & $2.168(1.567-2.998)$ & $<0.0001$ \\
\hline & IIB/IIIA use & $0.802(0.586-1.098)$ & 0.17 \\
\hline
\end{tabular}

Abbreviations: CK-MB, creatinine kinase myocardial brain fraction; CPK, creatinine phosphokinase; IABP, intra-aortic balloon pump; IIB/IIIA, glycoprotein IIB/IIA inhibitor; LAD, left anterior descending artery; LVEF, left ventricular ejection fraction.

thrombectomy during PCI for STEMI neither benefitted patients, nor jeopardized myocardial salvage resulting in increased infarct size. ${ }^{7}$ Meta-analysis done by Bavry et al summarized the differential role of thrombectomy techniques on the outcomes. ${ }^{8}$ Another study analyzing nine clinical trials found that manual thrombectomy decreased distal embolization and lowered 30-day mortality. ${ }^{9}$ A meta-analysis done by Burzotta et al found that mortality and major adverse cardiac events were reduced only in patients treated with glycoprotein IIb/IIla inhibitors. ${ }^{10}$ Surprisingly, time to reperfusion and initial TIMI flow did not have any impact on the benefit of thrombectomy. Mongeon concluded after a metaanalysis of 21 clinical trials that routine thrombectomy did not improve 30-day mortality. ${ }^{11}$ The MUSTELA (MUltidevice thrombectomy in acute ST-segment Elevation Acute myocardial infarction) trial did not show any reduction in infarct size and transmurality as assessed by cardiac magnetic resonance imaging at 3 months in patients with high thrombus load. 
26 Thrombus Aspiration in Acute Myocardial Infarction Jaiswal et al.

Table 4 Death and reinfarction rates according to thrombectomy after PCI in patients with ST-segment elevation myocardial infarction

\begin{tabular}{|l|l|l|l|}
\hline \multirow{2}{*}{ Variable } & \multicolumn{2}{|l}{ Thrombectomy } & \multirow{2}{*}{$p$-Value } \\
\cline { 2 - 3 } & No (139) & \multicolumn{2}{l}{} \\
\hline Death & $2(1.4 \%)$ & $12(3.6 \%)$ & 0.09 \\
\hline In-hospital mortality/1 mo mortality & $5(3.6 \%)$ & $3(1 \%)$ & 0.04 \\
\hline 1 -y mortality & $3(2.2 \%)$ & $2(0.6 \%)$ & 0.15 \\
\hline$>1$ y mortality &
\end{tabular}

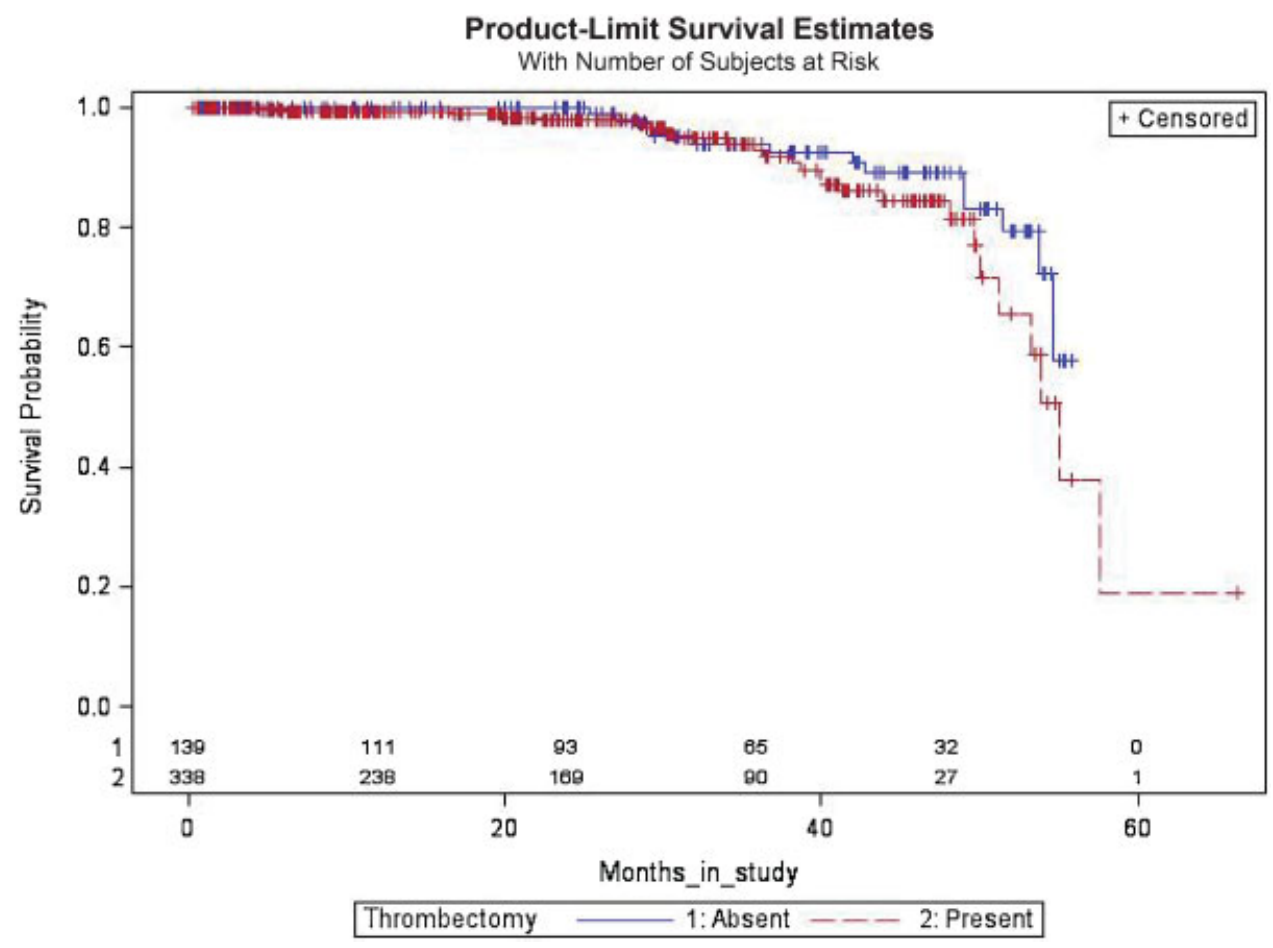

Fig. 1 Kaplan-Meier curves for survival rate comparing thrombectomy with conventional PCl group. The number of patients at risk is shown above the $x$-axis. Log-rank $p=0.35$. PCl, percutaneous intervention.

Most recent study, the TASTE (Thrombus Aspiration during ST-Elevation myocardial infarction) also concluded that manual thrombus aspiration before PCI had no significant effect on the primary endpoint of all-cause mortality at 30 days. The neutral outcome was consistent in all patient subgroups, regardless of baseline clinical or angiographic characteristics. $^{31}$

Routine thrombectomy is not frequently performed in the United States, but there is an increasing temporal trend. An analysis of the CathPCI registry data showed that thrombectomy was performed in only $19 \%$ of STEMI patients between July 2009 and December $2010 .^{32}$ Use of manual thrombectomy were more likely in patients with preprocedural TIMI 0/1 flow, younger age, saphenous vein graft, glycoprotein IIB/IIIA inhibitor use, and single vessel disease. Similar results were observed in our data, only patients with anterior infarction (OR: 1.7), preprocedural TIMI 0/1 flow (OR: 8.6), and IABP use (most likely for hemodynamic instability or decompensated heart failure) (OR: 0.4) were more likely to undergo thrombectomy. The difference in results regarding graft and GP IIB/IIIA inhibitor utilization could be attributed to lower rates of graft-related infarction and glycoprotein IIB/IIIA use in our cohort. Despite, the interventional cardiologists bias toward using thrombectomy in TIMI 0/1 flow patients, we did not find a significant interaction between preprocedural TIMI flow and the impact of thrombectomy on mortality or reinfarction rates. Postprocedural TIMI 3 flow was also similar between groups. This could be partly explained by improvements in the management of STEMI patients by the introduction of a newer generation of drug eluting stents, or the use of better pharmacotherapy including the early introduction of statins, angiotensin-converting enzyme inhibitors, optimal dual antiplatelet therapy, and cardiac rehabilitation.

Our study has several limitations. First, it represents a single-center experience using retrospectively collected 
data. However, our data set is representative of day-to-day community practices for the management of STEMI. Second, while the study population is modest in size, it remains comparable to several major studies undertaken. 2,3,5,6 Nevertheless, mortality was seen in only 30 patients. The patients with failed revascularization and those who died before completion of revascularization were excluded due to lack of data in our registry. This additional information might have affected the overall mortality and hence the interpretation. Third, we did not evaluate thrombus burden in these patients. However, the TAPAS trial ${ }^{3}$ randomized patients to thrombectomy irrespective of angiographic characteristics and findings from the TASTE trial also points toward a lack of association between thrombus characteristics and clinical outcomes. Finally, we were not able to include subsequent PCI, CABG, and nonfatal MI beyond 30 days due to lack of proper documentation. Despite these limitations, the results from this study are clinically relevant and should generate further discussion and motivate future prospective studies.

\section{Conclusion}

Our real-world observation of thrombectomy utilization, found that higher risk STEMI patients with hemodynamic instability are more likely to undergo thrombectomy during primary PCI in an uncontrolled clinical setting.

\section{Conflict of Interest}

None.

Funding

Current study is not supported by any research grant.

\section{References}

1 Sianos G, Papafaklis MI, Daemen J, et al. Angiographic stent thrombosis after routine use of drug-eluting stents in ST-segment elevation myocardial infarction: the importance of thrombus burden. J Am Coll Cardiol 2007;50(7):573-583

2 Srinivasan M, Rihal C, Holmes DR, Prasad A. Adjunctive thrombectomy and distal protection in primary percutaneous coronary intervention: impact on microvascular perfusion and outcomes. Circulation 2009;119(9):1311-1319

3 Svilaas T, Vlaar PJ, van der Horst IC, et al. Thrombus aspiration during primary percutaneous coronary intervention. $\mathrm{N}$ Engl J Med 2008;358(6):557-567

4 Vlaar PJ, Svilaas T, van der Horst IC, et al. Cardiac death and reinfarction after 1 year in the Thrombus Aspiration during Percutaneous coronary intervention in Acute myocardial infarction Study (TAPAS): a 1-year follow-up study. Lancet 2008; 371(9628):1915-1920

5 Sardella G, Mancone M, Bucciarelli-Ducci C, et al. Thrombus aspiration during primary percutaneous coronary intervention improves myocardial reperfusion and reduces infarct size: the EXPIRA (thrombectomy with export catheter in infarct-related artery during primary percutaneous coronary intervention) prospective, randomized trial. J Am Coll Cardiol 2009;53(4): 309-315
6 Brodie BR. Aspiration thrombectomy with primary PCI for STEMI: review of the data and current guidelines. J Invasive Cardiol 2010; 22(10, Suppl B):2B-5B

7 Kaltoft A, Bøttcher M, Nielsen SS, et al. Routine thrombectomy in percutaneous coronary intervention for acute ST-segment-elevation myocardial infarction: a randomized, controlled trial. Circulation 2006;114(1):40-47

8 Bavry AA, Kumbhani DJ, Bhatt DL. Role of adjunctive thrombectomy and embolic protection devices in acute myocardial infarction: a comprehensive meta-analysis of randomized trials. Eur Heart J 2008;29(24):2989-3001

9 De Luca G, Dudek D, Sardella G, Marino P, Chevalier B, Zijlstra F. Adjunctive manual thrombectomy improves myocardial perfusion and mortality in patients undergoing primary percutaneous coronary intervention for ST-elevation myocardial infarction: a metaanalysis of randomized trials. Eur Heart J 2008;29(24):3002-3010

10 Burzotta F, De Vita M, Gu YL, et al. Clinical impact of thrombectomy in acute ST-elevation myocardial infarction: an individual patient-data pooled analysis of 11 trials. Eur Heart J 2009;30(18):2193-2203

11 Mongeon FP, Bélisle P, Joseph L, Eisenberg MJ, Rinfret S. Adjunctive thrombectomy for acute myocardial infarction: A bayesian metaanalysis. Circ Cardiovasc Interv 2010;3(1):6-16

12 van 't Hof AW, Liem A, Suryapranata H, Hoorntje JC, de Boer MJ, Zijlstra F; Zwolle Myocardial Infarction Study Group. Angiographic assessment of myocardial reperfusion in patients treated with primary angioplasty for acute myocardial infarction: myocardial blush grade. Circulation 1998;97(23):2302-2306

13 Varbella F, Gagnor A, Luceri S, et al. Primary angioplasty and routine utilization of thrombus aspiration devices: feasibility and results in a consecutive series of 486 patients. J Cardiovasc Med (Hagerstown) 2007;8(4):258-264

14 Mangiacapra F, Wijns W, De Luca G, et al. Thrombus aspiration in primary percutaneous coronary intervention in high-risk patients with ST-elevation myocardial infarction: a real-world registry. Catheter Cardiovasc Interv 2010;76(1):70-76

15 Beaudoin J, Dery JP, Lachance P, et al. Impact of thrombus aspiration on angiographic and clinical outcomes in patients with STelevation myocardial infarction. Cardiovasc Revasc Med 2010; 11(4):218-222

16 Lemesle G, Sudre A, Bouallal R, et al. Impact of thrombus aspiration use and direct stenting on final myocardial blush score in patients presenting with ST-elevation myocardial infarction. Cardiovasc Revasc Med 2010;11(3):149-154

17 Noman A, Egred M, Bagnall A, Spyridopoulos I, Jamieson S, Ahmed J. Impact of thrombus aspiration during primary percutaneous coronary intervention on mortality in ST-segment elevation myocardial infarction. Eur Heart J 2012;33(24):3054-3061

18 O'Gara PT, Kushner FG, Ascheim DD, et al; American College of Emergency Physicians; Society for Cardiovascular Angiography and Interventions. $2013 \mathrm{ACCF} / \mathrm{AHA}$ guideline for the management of STelevation myocardial infarction: a report of the American College of Cardiology Foundation/American Heart Association Task Force on Practice Guidelines. J Am Coll Cardiol 2013;61(4):e78-e140

19 Steg PG, James SK, Atar D, et al; Task Force on the management of ST-segment elevation acute myocardial infarction of the European Society of Cardiology (ESC). ESC Guidelines for the management of acute myocardial infarction in patients presenting with ST-segment elevation. Eur Heart J 2012;33(20):2569-2619

20 Eeckhout E, Kern MJ. The coronary no-reflow phenomenon: a review of mechanisms and therapies. Eur Heart J 2001;22(9):729-739

21 Kushner FG, Hand M, Smith SC Jr, et al. 2009 focused updates: ACC/ AHA guidelines for the management of patients with ST-elevation myocardial infarction (updating the 2004 guideline and 2007 focused update) and ACC/AHA/SCAI guidelines on percutaneous coronary intervention (updating the 2005 guideline and 2007 focused update) a report of the American College of Cardiology Foundation/American Heart Association Task Force on Practice Guidelines. J Am Coll Cardiol 2009;54(23):2205-2241 
22 Migliorini A, Stabile A, Rodriguez AE, et al; JETSTENT Trial Investigators. Comparison of AngioJet rheolytic thrombectomy before direct infarct artery stenting with direct stenting alone in patients with acute myocardial infarction. The JETSTENT trial. J Am Coll Cardiol 2010;56(16):1298-1306

23 Stone GW, Webb J, Cox DA, et al; Enhanced Myocardial Efficacy and Recovery by Aspiration of Liberated Debris (EMERALD) Investigators. Distal microcirculatory protection during percutaneous coronary intervention in acute ST-segment elevation myocardial infarction: a randomized controlled trial. JAMA 2005;293(9): 1063-1072

24 Ali A, Cox D, Dib N, et al; AIMI Investigators. Rheolytic thrombectomy with percutaneous coronary intervention for infarct size reduction in acute myocardial infarction: 30-day results from a multicenter randomized study. J Am Coll Cardiol 2006;48(2): 244-252

25 Napodano M, Pasquetto G, Saccà S, et al. Intracoronary thrombectomy improves myocardial reperfusion in patients undergoing direct angioplasty for acute myocardial infarction. J Am Coll Cardiol 2003;42(8):1395-1402

26 Antoniucci D, Valenti R, Migliorini A, et al. Comparison of rheolytic thrombectomy before direct infarct artery stenting versus direct stenting alone in patients undergoing percutaneous coronary intervention for acute myocardial infarction. Am J Cardiol 2004; 93(8):1033-1035
27 Lefèvre T, Garcia E, Reimers B, et al; X AMINE ST Investigators. $\mathrm{X}$-sizer for thrombectomy in acute myocardial infarction improves ST-segment resolution: results of the X-sizer in AMI for negligible embolization and optimal ST resolution (X AMINE ST) trial. J Am Coll Cardiol 2005;46(2):246-252

28 Burzotta F, Trani C, Romagnoli E, et al. Manual thrombus-aspiration improves myocardial reperfusion: the randomized evaluation of the effect of mechanical reduction of distal embolization by thrombus-aspiration in primary and rescue angioplasty (REMEDIA) trial. J Am Coll Cardiol 2005;46(2):371-376

29 De Luca G, Suryapranata H, Chiariello M. Aspiration thrombectomy and primary percutaneous coronary intervention. Heart 2006;92(7):867-869

30 Silva-Orrego P, Colombo P, Bigi R, et al. Thrombus aspiration before primary angioplasty improves myocardial reperfusion in acute myocardial infarction: the DEAR-MI (Dethrombosis to Enhance Acute Reperfusion in Myocardial Infarction) study. J Am Coll Cardiol 2006;48(8):1552-1559

31 Fröbert O, Lagerqvist B, Olivecrona GK, et al; TASTE Trial. Thrombus aspiration during ST-segment elevation myocardial infarction. N Engl J Med 2013;369(17):1587-1597

32 Owan TE, Roe MT, Messenger JC, Dai D, Michaels AD. Contemporary use of adjunctive thrombectomy during primary percutaneous coronary intervention for ST-elevation myocardial infarction in the United States. Catheter Cardiovasc Interv 2012;80(7):1173-1180 